\title{
Microsatellite Flyby to the Moon's South Pole
}

\author{
P. Dente, E. Castanho, F. Rosa, F. Oliveira, G. Ribeiro \\ Departamento de Ciências Aeroespaciais \\ Universidade da Beira Interior \\ Covilhã, Portugal
}

\begin{abstract}
Small satellites have become one of the most relevant technologies nowadays, however their purpose is mostly academic. This paper summarizes a project of a CubeSat that begins its journey on a Geostationary Transfer Orbit (GTO) and ends up performing flybys to the Moon's south pole, aiming to gather observations of lunar craters, in order to find iced water crystals. This mission gives an innovative and low-cost solution for an interplanetary mission, demonstrating the capability of CubeSats.
\end{abstract}

Keywords-CubeSat; Moon; NANOSTAR; Student Design Challenge

\section{INTRODUCTION}

In the late 1990s, a spacecraft called Lunar Prospector sensed large amounts of hydrogen at the Moon's poles. After two years, NASA developed the Lunar Crater Observation and Sensing Satellite (LCROSS) mission that reached out and touched the hydrogen detected on the Moon. The lunar soil was analysed, and it was proven that there are, in fact, ice crystals of water on the Moon's poles [1].

With the growing interest of sending humans to the surface of Mars, finding big quantities of water on the Moon would allow the creation of an important lunar outpost [2].

Past and future missions such as LunaH-Map, Lunar IceCube, Lunar Flashlight and LunIR provided important data during the project presented in this paper.

This paper is organized as follows: section II describes all the subsystems of the satellite. In section III, the risk analysis and mitigation are introduced. Finally, the conclusions about this project are presented in section IV.

\section{Mission ANALYSIS AND SATELLITE DESIGN}

\section{A. Mission Analysis}

The mission designed has 7 different phases. Firstly, the satellite is placed in the desired orbital location from its initial position. Then, one loop sequence is established with the objective of raising the orbit apogee and approach the satellite to the lunar orbit. After the loop sequence, the satellite must be placed in the desired orbital location to start the lunar transfer that will place the satellite in the Moon's south pole, performing the first flyby. Then, it will perform one orbit change and it will propagate to the new orbit apogee where it will start the manoeuvre to perform a second and closer flyby.

\author{
J. Benedicto \\ ETSIAE \\ Universidad Politécnica de Madrid \\ Madrid, Spain
}

After this last orbit change, the satellite will orbit the Moon and begin transferring data to Earth.

The total Delta-V required with margin, is around $1319 \mathrm{~m} / \mathrm{s}$.

The trajectory of the mission was carefully analyzed using the General Mission Analysis Tool (GMAT).

\section{B. Payload}

A camera is used to take pictures of the lunar surface; however, it is not enough to check whether the hydrogen found on a specific crater on the Moon is in the form of water $\left(\mathrm{H}_{2} \mathrm{O}\right)$ or hydroxyl $(\mathrm{OH})$. So, one needs to add spectrometers to the payload that examine light emitted or absorbed by materials to help identify their composition. The Neutron Spectrometer System (NSS) and the Near-Infrared Volatile Spectrometer System (NIRVSS) are the spectrometers used.

\section{Space Propulsion Subsystem}

The selected propulsion system is the BGT-X5, a green monopropellant chemical thruster designed by Busek. This thruster operates with AF-M315E, a very stable propellant that provides $10 \%$ higher Isp, and $45 \%$ greater density than the highly toxic hydrazine, therefore allowing a smaller and lighter propellant tank. The system has $1 \mathrm{U}$ volume and can be scaled by adjusting the size of the propellant tanks. With nearly $225 \mathrm{~s}$ of specific impulse and $500 \mathrm{mN}$ of thrust, the estimated time to reach the Moon is of approximately 42-43 days. Given the calculated Delta-V, and applying the Tsiolkovsky equation, the estimated propellant budget is $6.6 \mathrm{~kg}$.

\section{Attitude, Determination and Control Subsystem (ADCS)}

This subsystem is composed of a Cube ADCS 3-axis Integrated Unit (IU) from CubeSatShop that combines different attitude and navigation components into a single part with the aim to provide a simple solution to the satellite's requirements [3]. Some components of this IU include reaction wheels with pointing accuracy of $8.727 \mathrm{e}-3 \mathrm{rad}\left(0.5^{\circ}\right)$, gyroscopes and accelerometers.

The IU has a Cube Computer module, which is used as main on-board computer and main storage (2GB) for the data generated by the camera.

\section{E. Communications Subsystem and Ground Segment}

The CubeSat X-band patch antenna from EnduroSat is used in this subsystem. It has operating frequency between 
$8025 \mathrm{MHz}$ and $8400 \mathrm{MHz}$ and radio frequency output power up to $4 \mathrm{~W}$. From the same manufacturer it was chosen the X-band transmitter with the same frequency range.

The NSS (New Space Systems) GPS Receiver from CubeSatShop allows a more reliable transmission signal, with knowledge of time and other features.

The final link budget for the satellite is $33.7 \mathrm{~dB}$, which will be established by a ground station from ESA located $77 \mathrm{~km}$ west of Madrid, Spain.

The satellite's antenna transmission speed is $10000 \mathrm{bps}$, so to transmit the estimated $10 \mathrm{MB}$ of data of each flyby one will need about 8400s. According to GMAT, each contact periods, while orbiting the Moon, will be around 40000s, so each transmission will be done easily after each flyby.

\section{F. Electric Power Subsystem (EPS)}

For the satellite designed in this paper the average power is $36 \mathrm{~W}$ and the peak power is $51 \mathrm{~W}$, these values are in excess. One must size a triple junction Gallium-Arsenide (GaAs) photovoltaic system using the above power budget values, GMAT, constants from the literature and equations from [4].

The solar panel needs to provide $70 \mathrm{~W}$ during daylight for the entire orbit, which is equivalent to a total area between $0.14 \mathrm{~m}^{2}$ and $0.21 \mathrm{~m}^{2}$. The Custom PMDSAS panel from Pumpkin SpaceSystems is a great choice, because it has coarse sun and external temperature sensors include, and each cell provides $1 \mathrm{~W}$, so to get $70 \mathrm{~W}$ one needs 72 cells for symmetry.

Energy storage is important to provide backup power for long missions. The ideal battery capacity for the satellite presented in this paper is $46.71 \mathrm{Wh}$ to $93.42 \mathrm{Wh}$, being the Intelligent Protected Lithium Battery from Pumpkin SpaceSystems a good option.

To control and regulate the power in all subsystems it is used the NanoPower P60 from GOMspace.

\section{G. Mechanical Design and Structure}

After several iterations, a 6U configuration was obtained which allows to accommodate all the satellite's components with $14 \mathrm{~kg}$ of total mass. The structure from EnduroSat is a good choice.

More than half of the satellite's volume is for the chemical propulsion system, because it requires a large amount of propellant to reach the Moon.

When the satellite is executing a flyby, a sliding door will open, exposing the camera and the spectrometers. The ADCS is located on the centre of gravity, so that the reaction wheels perform less effort during manoeuvres.

Inside the satellite, there are gimbals that fold the solar arrays in order to retract them to the satellite's external structure. The solar arrays can reach high temperatures, so they must be placed away from the payload and other subsystems.

The antenna is placed in the opposite side of the payload, so it can be more easily pointed towards Earth after each flyby.

\section{H. Thermal Control Subsystem}

The Multi-Layer Insulation (MLI) is a well-known type of passive thermal method that is used to reduce the heat exchanges by radiation between the external surfaces of the satellite and the environment. The MLI consists of several layers, where the surfaces turned to the space and the satellite are made of Kapton and the layers between the two surfaces are made of Mylar, which is highly resistant and dimensionally stable by high temperature action.

\section{RISK ANALYSIS AND MitiGATION}

The damage of electronic components due to Van Allen radiation belts has a very high probability, however with the use of component redundancy it only represents a very low impact.

If the altitude of the first flyby exceeds the required margins for the second one, it will be necessary a more profound trajectory analysis, this represents a medium probability and impact.

If the satellite is unable to transmit data to Earth, it will attempt to communicate with another ESA ground station. The ADCS failure can be overcome with redundancy, e.g. more reaction wheels. These two risks have a very low probability with high impact.

The thruster failure has a very high impact on the mission and cannot be mitigated, however the probability is very low.

Battery cell failure has low probability and impact because of the high redundancy ( 8 cells).

\section{CONCLUSIONS}

The preliminary design gives an important path that must be followed regarding the satellite project, where an iterative process must be done in order to get the final optimal solution.

The satellite mission that was given in this paper resulted in the winning solution for the NANOSTAR Project.

\section{ACKNOWLEDGMENT}

This work was supported by the professors from the NANOSTAR Project and Jorge Monteiro. The authors would also like to thank Universidade da Beira Interior (UBI) for sponsoring the trip to the United Kingdom.

NANOSTAR Project is funded by the Interreg Sudoe Programme through the European Regional Development Fund (ERDF). ERDF budget: 1,547,184.75 €.

\section{REFERENCES}

[1] NASA, "What is LCROSS, the Lunar Crater Observation and Sensing Satellite?," 2019. [Online]. Available: https://www.nasa.gov/ames/LCROSS. [Accessed: 29-Jul-2019].

[2] NASA, "Moon to Mars Overview," 2019. [Online]. Available: https://www.nasa.gov/topics/moon-to-mars/overview. [Accessed: 29Jul-2019].

[3] NASA, "Small Spacecraft Technology State of the Art," 2015.

[4] J. Wertz, D. Everett, and J. Puschell, Space Mission Engineering: The New SMAD. Microcosm Press, 2011. 